\title{
Design of Flexible Platform for Various Free-Space, Broadband Dielectric Analysis Measurement Techniques.
}

\author{
Yaqiang Liu ${ }^{(1)}$ and Dr. Paul. O’Leary ${ }^{(1)}$ \\ (1) Waterford Institute of Technology, Waterford, Ireland \\ E-mail:20018753@mail.wit.ie
}

\begin{abstract}
Non destructive testing (NDT) is an important analysis technique to understand material composition. Dielectric analysis is an NDT technique, using a probing electromagnetic wave, which is reflected and/or propagated from/through the material under test (MUT). There have been many advances in this area, with several frequency domain techniques based on measurement of amplitude and phase, amplitude alone or phase alone. This paper describes the design of a new dielectric analysis test platform, which includes controllable variable widths of MUT, antenna translation and even accurate angular positioning. This test platform will allow comparison of several of the recognised techniques whose various aims include to lower cost, to remove diffraction and multiple reflection effects. In our case, the target dielectrics are in powder or liquid form. The proposed test platform is a physical extension of an existing test platform, developed to qualify propagation reduction for microwave signals passing through energy efficient glass, coated with transparent conductors.
\end{abstract}

\section{Introduction}

Non-destruction material characterisation can be performed by measuring their complex permittivity and permeability. Dielectric analysis has advanced considerably and now includes several frequency and time domain techniques, in free space and also confined space, for individual frequencies and also broadband, for both high and low loss materials [refs]. Developments have included a reduction of the effects of multiple reflections, of diffraction from boundary edges, of initial guesses for $\varepsilon_{r}$, of precise sample thickness knowledge, and of phase uncertainty in reflection measurements [refs ]. Many of the free space techniques use or could be used on similar platforms and this concept forms the basis of the test platform design described in this work.

Free space techniques offer several advantages $\mathrm{xx}$ a wide dynamic range for measurements. Reflection only free-space measurement techniques are possible, to obtain the dielectric properties from the reflection coefficients, and consists of an antenna illuminating a flat-surface MUT. Although simple, such techniques can suffer Xxx

, techniques that include propagation measurements offer the advantages of reduced impact of surface roughness (at higher frequencies), local variations in material composition can be averaged out and standard calibration techniques can be applied. The proposed platform should accommodate reflection only, as well as transmission/reflection techniques. It will also be flexible in accommodating various sample thicknesses to overcome.... 
One challenge, addressed in some techniques relates to low- or medium-loss materials, where the measured $S_{21}$ and $S_{11}$ do not yield a unique solution if the material has a long electrical length.

It would be interesting to examine several of these techniques in the same environment, for the same materials, by using this same platform.

\section{Research Background}

We developed a test platform originally to qualify propagation through energy efficient glass windows, where the energy efficiency was attained through both double glazing and also by coating one of the glass panes with a transparent conductor [ref Lucca, RIA, WIT]. Both propagation and reflection measurements at standard Radio Frequency (RF) communication frequencies were required to qualify window performance. Subsequently, the setup was modified, through the addition of a bespoke tank, to facilitate the dielectric analysis of powders and liquids. The tank fit into the original structure and had a fixed, inflexible design. [Joe, should we include a picture here?].

\section{Measurement Methods xx}

The tests for complex dielectric properties are carried out using the bi-directional Rohde \& Schwarz vector network analyser (VNA) ZVB-20. This means the usual VNA S-parameter measurements can be taken, as well as simply taking amplitude or phase measurements. Various techniques to convert free-space S-parameters measurements into dielectric properties have been proposed. In early 1966, R. M. Redheffer [1] was the first researcher to suggest a simple free-space method for measurement of dielectric constant from the measured phase of transmission coefficient. He reported that free-space methods are non-destructive and contactless techniques which are specially suited for dielectric measurement of materials. In 1971, Harold L. Basset [2] was the first researcher to measure complex permittivity in free-space using spot-focusing antennas at a frequency of 9.4 GHz. He measured complex permittivity of fused silica as a function of temperature from ambient to $2500 \mathrm{C}$.

\section{Test Platform Design}

The design aims to have a linear time invariant system, with source stability ensured by the VNA, addressing: diffraction effects from edges of samples and interfering reflections from the surrounding environment, ground reflections and measurement system losses through calibration, absorbers on the ground, height selection of antennas, appropriate shielding of antennas from external interference and a spectrum analyser 'sniffer' to confirm the absence of interference.

Given that several techniques perform better when measurements are made over various thicknesses (at least two and in some cases three, first proposed in [Contribution]), that calibration should occur at least one container face and that measurements of the container wall S-parameters can also be required, the new design consists of glass panes that can be individually set into various positions in 
the holding structure. Spacing between panes is achieved through appropriate spacers. Given the flexible spacing, we can assume that the MUT can either be made electrically thin or, for wide spacing, has sufficient material loss that the multiple reflections between the two surfaces of the sample can be neglected. Glass is reasonably rigid, but to reduce the effects of sample thickness variation, material thicknesses can be varied on occasion up to $18 \mathrm{~cm}$. As powders and liquids are the target materials, known issues such as composition variation, in measuring two or three sample thicknesses, should be less of an issue, especially if care is taken to ensure no air pockets. Powder could of course be measured using a resonant cavity, but not broadband. On the other hand, non-resonant waveguide transmission/reflection methods would require much less material than a free-space technique and are therefore the most used broadband technique.xx

Measured reflection or transmission/ reflection scattering $(S$-) parameters have been used for broadband permittivity measurement. The elimination of errors due to multiple reflections using the TRL (thru, reflect, line) $[5,6]$ and the dual separation [7] calibration techniques will be facilitated. The TRL, however, is only applicable when both reflection and transmission measurements are performed on the sample.

The test platform, originally developed for glass propagation tests, is in a big hall (approximately $45 \mathrm{~m} \times 30 \mathrm{~m} \times 8 \mathrm{~m}$ ) (Figure 1). The propagation properties are measured in the far field, according to the conventional $2 \mathrm{D}^{2} / \lambda$, which for the selected Schwarzbeck horn antenna BBHA 9120, D=0.33m. Thus the minimum far field separation, at the maximum test frequency of $6 \mathrm{GHz}$, is $4.35 \mathrm{~m}$ [3]. In addition, for higher frequency measurement, a second set of antennas is introduced, which are the Schwarzbeck Horn antenna JXTXLB-90-20-C 8.2GHz to $12.4 \mathrm{GHz}, \mathrm{D}=0.27 \mathrm{~m}$. However, under qualification tests the Standing Wave Ratio for the latter antenna showed a higher usable bandwidth, which is $7.5 \mathrm{GHz}$ to $15 \mathrm{GHz}$, taking the usable bandwidth as the region where the $\mathrm{SWR} \leq 2$. But, at $15 \mathrm{GHz}$, the far field distance is the considerable separation of $11 \mathrm{~m}$ (should this be 7.2m??)[3]. The antennas are mounted on a modified Pentax Precision Total Station [4] (Figure 1), which permitted accurate distance and angular measurements, as well as movement of the antenna along the axis of propagation to optimize received signal strength, when required.

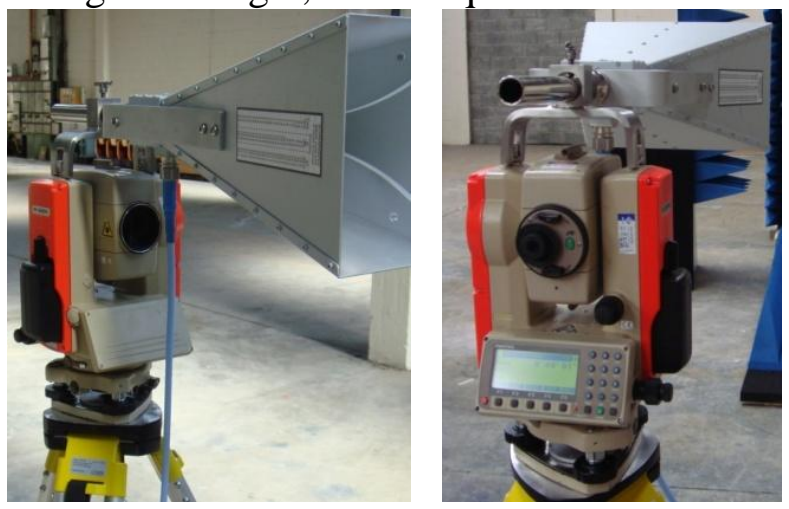

Figure 1Modified total station antenna mount 
The measurements will be performed with a Rohde \& Schwarz ZVB-20 Vector Network Analyser (VNA). The Rhode \& Schwarz calibration kit ZV-Z32 is used for the cable calibrations. The cables chosen are 7.62m long Sucoflex 106, lowloss cables from Huber + Suhner, which are sufficiently long to allow relatively large antenna separations if needed.

A glass tank is developed for early non solids material test, albeit with a fixed thickness.

\section{Measurement Result and Analysis}

The first preliminary result is done for the two layers, $4 \mathrm{~mm}$ normal glass. The distance, in this case, between the two antennas is $5 \mathrm{~m}$, so the frequency range is from $800 \mathrm{MHz}$ to $6 \mathrm{GHz}$, in order to comply with the far field requirements. All measurements are presented relative to free space and consequently phase and attenuations represent changes due to the MUT.

The measurement is also applied to several non solids materials, such as salt, sugar and tap water. The sample materials are sandwiched in the glass tank. For different dielectric test materials, the electrical length is unknown beforehand, complicating the dielectric constant calculation. This research work aims to develop a measurement structure to overcome this difficulty, by replicating the first measurement over a slightly thicker test sample, where the thickness is flexibly altered in a controlled manner. By measuring the transmission and reflection coefficients of the sample over both thicknesses, we can obtain the electrical length in the sample material, and furthermore, the dielectric constant of this material can be defined. This leads to a new design of the glass tank, with a flexible thickness.

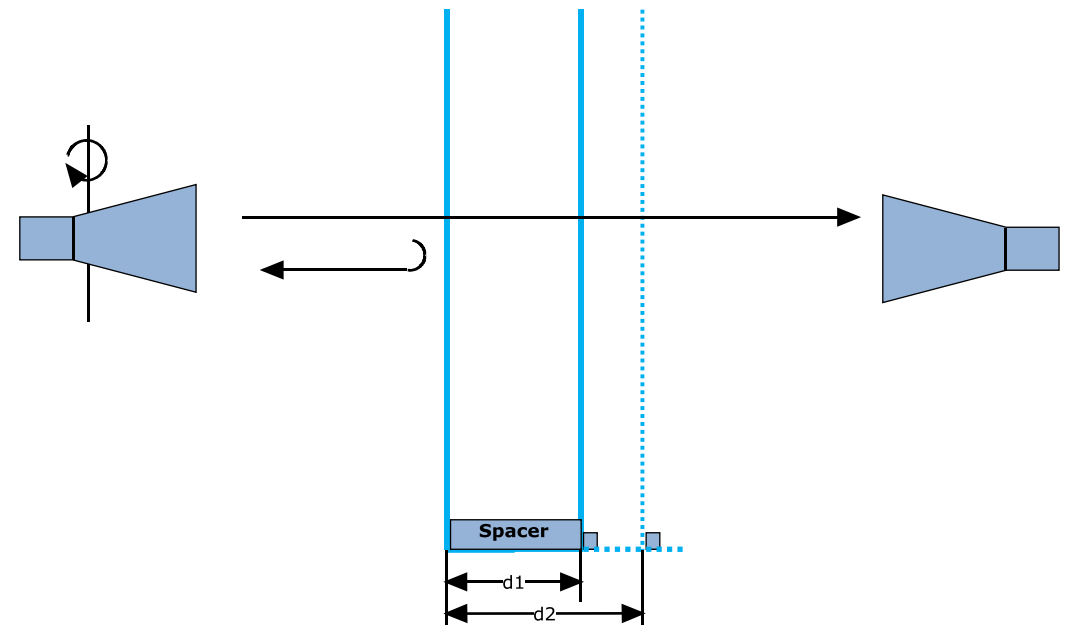

Figure 2 Next generation glass tank, with a flexible thickness 


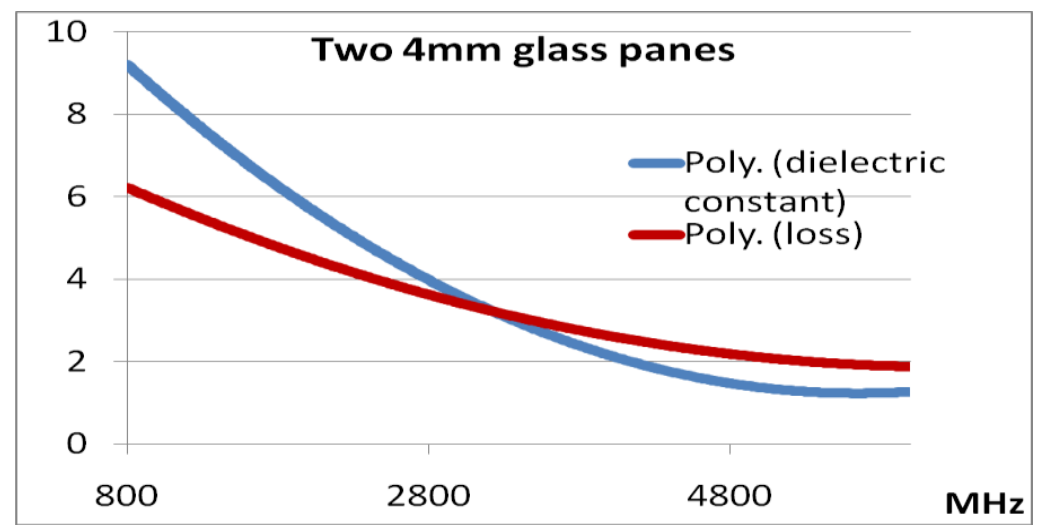

Figure $38 \mathrm{~mm}$ glass dielectric constant

\section{Calculation Algorithm}

The reflection and transmission coefficients are related to the scattering parameters and given by:

$$
\begin{aligned}
& S_{11}=\frac{\Gamma\left(1-T^{2}\right)}{1-\Gamma^{2} T^{2}} \\
& S_{21}=\frac{T\left(1-\Gamma^{2}\right)}{1-T^{2} \Gamma^{2}}
\end{aligned}
$$

Those parameters can be easily obtained from vector network analyser The reflection coefficient, $\Gamma$, is given by

$$
\Gamma^{\prime \prime}=\mathrm{X} \pm \sqrt{\left(\mathrm{X}^{2}-1\right)}
$$

where

$$
\mathrm{X}=\frac{\mathrm{S}_{11}^{2}-\mathrm{S}^{2}+1}{2 * \mathrm{~S}_{11}}
$$

The transmission coefficient, $\mathrm{T}$, is:

$$
\mathrm{T}=\frac{\mathrm{S}_{11}+\mathrm{S}_{21}-\Gamma}{1-\left(\mathrm{S}_{11}+\mathrm{S}_{21}\right) \Gamma}
$$

The chosen of the plus or minus is defined by $|\Gamma|<1$.

Then we can obtain

$$
\begin{aligned}
& \varepsilon=\frac{\gamma}{\gamma_{0}}\left(\frac{1-\Gamma}{1+\Gamma}\right) \\
& \mu=\frac{\gamma}{\gamma_{0}}\left(\frac{1+\Gamma}{1-\Gamma}\right)
\end{aligned}
$$

The propagation constant can be written as

$$
\gamma=\left[\log _{e}(1 /|\mathrm{T}|)\right] / d
$$

Where $d$ is the thickness of material under test.

Since $T$ is a complex number, the equation can be re-written as

Where $n=0, \pm 1, \pm 2, \ldots$

$$
\gamma=\left[\log _{\theta}(1 /|\mathrm{T}|)\right] / d+j\left(\frac{2 \pi n+\phi}{d}\right)
$$


The real part is single valued, but the imaginary part may have multiple values. So,

$$
\begin{aligned}
& \text { imaginary part of } \gamma=\text { phase constant }(\beta)=2 \pi / \lambda_{m} \\
& \qquad \frac{d}{\lambda_{m}}=n+\frac{\phi}{2 \pi}
\end{aligned}
$$

- When $n=0$ and $0<\phi<2 \pi, d / \lambda_{m}$ is between 0 and 1. If the thickness $d$ is less than $\lambda_{m}$, there will be a unique value for the complex permittivity and permeability.

- When $d>\lambda_{m}$, one solution is to make the measurements on two different thicknesses of the material under tests.[5]

Equation (11) is the key to solving for propagation in a MUT. However, solving the imaginary part presents a challenge, unless the material width, $d$, happens to be smaller than the material wavelength, $\lambda_{m}$. This is difficult to guarantee, even for low values of permittivity, over the range of frequencies tested so far (wavelength in air falls from $37.5 \mathrm{~cm}$ down to $5 \mathrm{~cm}$ ). As the future work will also include higher frequencies (wavelength in air ranging from $5 \mathrm{~cm}$ down to $1.5 \mathrm{~cm}$ ), it is then impossible to guarantee this condition.

For any material thickness, $d_{i}$, the measured phase change, $\phi_{i}$, and the material wavelength, $\lambda_{m}$, are related by:

$$
d_{i}=n_{i} \lambda_{m}+\frac{\phi_{i}}{2 \pi} \lambda_{m}, 0<\phi_{i}<2 \pi
$$

The material thickness, $d_{i}$, and the phase change, $\phi_{i}$, can be measured, but neither the material wavelength, $\lambda_{m}$, nor the number of wave periods in the material, $n_{i}$, are known. Both are required in the solution of equation (12).

The future work proposed for this thesis will include control variation in the thickness $d_{i}$, when measuring the dielectric response of powders and liquids. This controlled increase will also allow the experimenter to avoid a situation where the new thickness is an integer multiple of material wavelengths more than the previous thickness. Clearly this will then yield two linear equations, where the thicknesses and phase changes can be measured, leading to an easy solution for the material wavelength, $\lambda_{m}$, and the number of wave periods in the material, $n_{i}$.

\section{References}

[1] R. M. Redheffer, "The measurement of dielectric constant," Techniques of Microwave Measurements, vol. 2, pp. 591-657, 1966.

[2] H. L. Bassett, "A free-space focused microwave system to determine the complex permittivity of materials to temperature exceeding $2000^{\circ} \mathrm{C}$," The Review of Scientific Instruments, vol. 42, pp. 200-204, 1971.

[3] Yaqiang Liu, "Different definations of Antenna far field boundary," Waterford Institute of Technology, Waterford, 2009.

[4] Ltd Pentax Precision Co. Introduction Manual Electronic Total Station R-300 Series. [Online]. http://www.geosolution.com/download/r300x-quick-basicenglish.pdf 
[5] D.K. Ghodgaonkar, V.V. Varadan and V.K. Varadan, "Free-space measurement of complex permittivity and complex permeability of magnetic materials at microwave frequencies," IEEE Trans. Instrum. Meas., vol. 39, pp. 387-394, April 1990.

[6] D. K. Ghodgaonkar, V. V. Varadan, and V. K. Varadan, "A free-space method for measurement of dielectric constants and loss tangents at microwave frequencies," IEEE Trans. Instrum. Meas., vol. 37, pp. 789-793, June 1989.

[7] Mansor Nakhkash, Yi Huang, Waleed Al-Nuaimy, and M. T. C. Fang An Improved Calibration Technique for Free-Space Measurement of Complex Permittivity, IEEE Trans on Geoscience and Remote Sensing, vol. 39, No. 2, Feb. 2001

Amplitude only, including flexible siting of receive antenna to maximise SNR:xx

[8] Hasar, U.C.; , "A Microcontroller-Based Microwave Measurement System for Permittivity Determination of Fresh Cement-Based Materials," Instrumentation and Measurement Technology Conference Proceedings, 2007. IMTC 2007. IEEE, vol., no., pp.1-6, 1-3 May 2007

M. Rodríguez-Vidal and E. Martín "Contribution to numerical methods for calculation of complex dielectric permittivities", Electron. Lett., vol. 17, p. 510, 1970.

[Improved] M. Nakhkash, Y. Huang, W. Al-Nuaimy, and M. Fang, "An improved calibration technique for free-space measurement of complex permittivity," IEEE Trans. Geo. Remote Sensing, vol. 39, no. 2, pp. 453-455, Feb. 2001. 